\title{
CoVID-19 containment: China provides important lessons for global response
}

\author{
Shuxian Zhang ${ }^{1, *}$, Zezhou Wang ${ }^{2, *}$, Ruijie Chang ${ }^{1}$, Huwen Wang ${ }^{1}$, Chen $\mathrm{Xu}^{1}$, Xiaoyue Yu${ }^{1}$, Lhakpa Tsamlag ${ }^{1}$, \\ Yinqiao Dong ${ }^{3}$, Hui Wang $(\bowtie)^{1}$, Yong Cai $(\bowtie)^{1}$ \\ ${ }^{1}$ School of Public Health, Shanghai Jiao Tong University School of Medicine, Shanghai 200025, China; ${ }^{2}$ Department of Cancer Prevention, \\ Shanghai Cancer Center, Fudan University, Shanghai 200032, China; ${ }^{3}$ Department of Environmental and Occupational Health, School of \\ Public Health, China Medical University, Shenyang 110122, China
}

(C) Higher Education Press and Springer-Verlag GmbH Germany, part of Springer Nature 2020

\begin{abstract}
The world must act fast to contain wider international spread of the epidemic of COVID-19 now. The unprecedented public health efforts in China have contained the spread of this new virus. Measures taken in China are currently proven to reduce human-to-human transmission successfully. We summarized the effective intervention and prevention measures in the fields of public health response, clinical management, and research development in China, which may provide vital lessons for the global response. It is really important to take collaborative actions now to save more lives from the pandemic of COVID-19.
\end{abstract}

Keywords coronavirus disease 2019 (COVID-19); control measure; public health response

\section{Background}

The novel coronavirus disease (COVID-19) is now fast spreading to 94 countries and, updated as of March 7, 2020, 101927 confirmed cases have been reported worldwide [1]. In China, with the unprecedented control measures, the numbers of newly confirmed and suspected cases reported have been declining on the whole over the past two weeks. On March 7, 2020, 44 new cases of confirmed infections were reported, with very few local community-based cases in China outside of Hubei Province [2]. The WHO-China Joint Mission report said that China has rolled out perhaps the most ambitious, agile, and aggressive disease containment efforts in history [3]. By striking contrast, the number of confirmed cases outside China is quickly climbing following an exponential growth trend. The total number of COVID-19 cases outside of China has reached 21110 including 413 deaths as of March 7, 2020 [1]. Forty-five countries already reported local transmission following importation. WHO raised the risk assessment of COVID-19 from "high" to

Received March 8, 2020; accepted March 9, 2020

Correspondence: Yong Cai, caiyong202028@hotmail.com; Hui Wang, huiwang@shsmu.edu.cn

*Shuxian Zhang and Zezhou Wang contributed equally to this manuscript. "very high" at a global level. China's approach to contain the spread of the virus has changed the trajectory of the epidemic [3]. China's efforts to contain the novel coronavirus can provide vital lessons for other nations experiencing the rapid spreading or at the risk of an epidemic.

\section{Public health response}

Shortly after the official announcement of the epidemic of COVID-19, the entire machinery of China started to take coordinated and comprehensive approaches for infectious disease control. The possible initial source of the novel coronavirus in China, the Huanan Seafood Wholesale Market, was shut down on January 1, 2020 [4]. On January 20, 2020, COVID-19 was included in the statutory reporting of Class B infectious diseases. Wuhan, the epicenter of the outbreak in China, was on lockdown since January 23, 2020 [5]. By January 26, 2020, 30 provinces in China had activated the first-level public health emergency response mechanism.

To prevent and control the spread of pneumonia caused by the novel coronavirus, China allocated an additional 9.95 billion CNY (about 1.44 billion USD) in funds for public health service and epidemic prevention and control [6]. The extension of the Spring Festival holiday, the postponed spring semester, the cancel of mass gathering 
activities and the control of transportation capacity were all aimed at reducing the movement of people.

Civil society organizations took responsibility of isolating residents in every community and helping solve practical life difficulties. At the individual level, home isolations, social distancing, and personal protection equipment such as face masks were implemented to prevent community transmission nationwide. Thanks to the development of advanced technology, residents have had easy access to reliable information and medical guidance, which can reduce misinformation and the impact of rumors. The public were well educated on the seriousness of COVID-19 to comply with the national approach of hand-washing, mask-wearing, social distancing, and universal temperature monitoring cooperatively. All people were keenly aware of their roles in preventing the virus from spreading.

To strike a balance between epidemic control and normal social and economic operations, industrial activities were gradually resuming in phases and batches since February 8, 2020 [7]. The supply of daily necessities was kept stable in every stage of the outbreak to ensure the smooth operation of society. The shortage of personal protective equipment (PPE) caused by rising demand was attenuated by mid-February 2020. By the end of February 2020 , the daily production capacity of masks in China exceeded 110 million units [8]. China is trying to resume normal social and economic activities with the maintenance of powerful containment strategy.

The prevention and control strategies were refined over time to fit in the complicated and changeable situations. A latest research estimated the epidemic trend in Wuhan, China using phase-adjusted SEIR model [9]. It provided estimation for epidemic trend after strict prevention and control measures implemented in China and predicted that the number of infections would decline in late February 2020 , in support of the outcome of epidemic: the outbreak could be effectively controlled soon [9]. The decline in COVID-19 cases across China has proved that the agile and stringent measures in multiple settings effectively interrupted transmission chains and reversed the escalating cases.

\section{Clinical management}

In the response to the unpredictable outbreak, healthcare resources including the dedication of medical workers as well as PPE and testing kits have all played important roles. In this ongoing severe epidemic, healthcare providers in China have treated patients with courage and conviction day and night. More than 40000 front-line medical workers have been mobilized from other regions of China to support Wuhan. Remarkably, two designated hospital and several mobile cabin hospitals were quickly established in Wuhan $[10,11]$. Bed capacity within Wuhan has increased to over 50000 [3]. All home-isolated patients were asked to be hospitalized in the newly built square cabin hospitals since February 2, 2020. In addition, all close contacts and suspected cases were demanded for mandatory isolation in special facilities and received medical observation for 14 days. Unprecedented measures were taken to ensure that all cases got treatment.

Also, with modern science and technology, a series of reliable and sensitive diagnostic tools were developed just several days after the first genomic sequence of the virus was released from Chinese research institutions. The speed was unimaginable even a few years ago. By March 5, 2020, 14 kits for detection of SARS-CoV-2 were approved in China [12]. Widely available kits made it possible to immediately detect cases. After being diagnosed, patients were treated according to the "Diagnostic and treatment protocol for Novel Coronavirus Pneumonia" [13]. The National Diagnosis and Treatment Guidelines (NDTG) has released its seventh edition within seven weeks by the China National Health Commission with the deep-going understanding of the disease. Besides supportive care and investigational treatments, intubation, invasive ventilation and extracorporeal membrane oxygenation (ECMO) equipment are applied to support the treatment of critically ill patients. To minimize mortality among critical cases, the principle of "Four Concentrations" (concentrating patients, medical resources, experts, and treatment into special centers) was applied. Also, allocation of medical supplies was well coordinated to prevent the infection in medical workers. As of March 4, 2020, 55404 cases have recovered from COVID-19 [2]. The principle of early identification, early isolation, early diagnosis, and early treatment has showed its effect.

\section{Research development}

In addition to medical staff in the front-line of diagnosis and treatment, Chinese researchers also have been joining the "battlefield" of epidemic prevention and control as quickly as possible. According to incomplete statistics, more than 200 COVID-19 original articles have been published worldwide, and nearly 100 articles, news, and commentaries have been published in prestigious journals (e.g., the Lancet, NEJM, Nature, Science, JAMA, and BMJ), among which more than $60 \%$ are from Chinese researchers. These efficient research results, as well as accumulated knowledge and experience in SARS and MERS, have provided crucial support in the fight against the epidemic. On January 5, 2020, the Wuhan Institute of Virology (WIOV), the Chinese Academy of Sciences, and the Wuhan Municipal Center for Disease Control and Prevention (CDC) were the first to identify the sequences of the pathogen SARS-CoV-2. On January 7, 2020, WIOV 
and China National CDC succeeded in isolating the viral strains [14]. On January 10, 2020, scientists from Shanghai and Wuhan took the lead in submitting the genome sequence of the new coronavirus (COVID-19) to the NCBI GenBank database for information sharing with the international community. In the following days, the Chinese CDC released the sequence of primers and probes for nucleic acid detection of the new coronavirus, and the National Pathogen Microbiological Resource Bank released the information of the first virus strain and its electron microscope photographs, providing important intelligence for the global response to the epidemic [15]. Through viral pathogens and epidemiological studies, the relationship between the source of SARS-CoV-2 and the known poultry and livestock was preliminarily excluded. It was suggested that bats were the most likely source by scientists from WIOV [16]. In terms of detection and diagnosis, 14 diagnostic detection reagents have been approved for marketing in China, and the Ministry of Science and Technology continuously promoted the development and application of efficient and rapid onsite detection products to further improve their sensitivity, specificity, and portability. In terms of animal models, mice, monkeys, and other animal models of COVID-19 infection have been successfully constructed, providing basic resources for the safety and efficacy evaluation of drugs and vaccines. Our medical staff has published the clinical characteristics and epidemiological characteristics of the COVID-19 patients in journals for many times in a timely manner, ranging from the first 41 confirmed cases $[17,18]$ to the latest 72314 cases [19]. Positive progress has also been made in drug screening and treatment protocol research. By the end of February 2020, nearly 300 clinical trials related to COVID-19 had been registered in the Chinese Clinical Trial Registry. Remdesivir, chloroquine phosphate, a variety of anti-HIV drugs, tocilizumab, patients' convalescent plasma, stem cell therapy, and traditional Chinese medicine treatment, etc. may all have therapeutic effects on COVID-19 [20]. Most strikingly, the convalescent plasma from recovered patients and the appropriate use of monoclonal antibody drug tocilizumab, targeting respectively the virus and the inflammatory factor (cytokine) storm, have been shown effective in treating severe cases [12]. Both agents have been included into the 7th NDTG. In addition, China has actively carried out the research and development of vaccine from five technical routes including inactivated vaccine, recombinant genetic engineering vaccine, adenovirus vector vaccine, nucleic acid vaccine (mRNA vaccine and DNA vaccine), and vaccines made from attenuated influenza virus vaccine vectors, which have entered the efficacy and safety research stage [12]. Epidemiologists also conduct epidemiological surveys to study transmission routes (finding the transmission route of human-to-human transmission) and establish prediction models to estimate the epidemic trend. Several groups reported various estimated reproductive number $\mathrm{R}$ and generated prediction for the early and post control phase of this outbreak, providing valuable basis for government decision-making [21,22]. To effectively respond to the new coronavirus pneumonia outbreak and further enhance the capacity of new emergent infectious diseases prevention and control, the National Natural Science Foundation of China launched a special grant for researchers. China is working hard for scientific prevention and control in response to major public health emergencies, to reduce their impact on China's economy and society, improve the national governance system and social management capabilities.

\section{Solidarity of international community is the weapon to win the war against COVID-19}

It is worrisome that the epidemics in some countries including the Republic of Korea, the Islamic Republic of Iran, and Italy are currently quite severe, demonstrating the very high infection ability of SARS-CoV-2. The surprising speed of the geographical expansion and increase in numbers of cases will overload clinical resources especially in many developing countries, particularly in those with weakened healthcare infrastructure due to repeated natural disasters or protracted armed conflict. To allow timely sharing with other countries of the most up-to-date information on COVID-19 control and prevention, a critical multilingual manual has been published in both English and French by the Chinese CDC [23]. The lesson from China is conveying a key message that the epidemic can be contained if the right measures are taken. We cannot ignore the fact that effective vaccine and antiviral therapeutics are still far from clinical application. Every nation needs to be ready to find and screen cases, isolate confirmed patients, trace close contacts, provide clinical treatment, prevent hospital outbreaks, and prevent community transmission. Although the collaborative public health actions taken in China have saved thousands of lives, the new imported confirmed cases from overseas is the new quiz to China. Flexible but powerful measures should be maintained to avoid the reemergence of the outbreak. As the virus does not respect borders and indiscriminately attacks people of all races, the solidarity and collaboration of all nations is the only way to fight against the virus. It is encouraging that a number of exchanges and collaborative projects between China, WHO, and many other countries have been conducted, including the recent sending of two volunteer expert teams by the Red Cross Society of China to Iran and Iraq in support of the control, diagnosis and treatment of epidemic. Chinese biomedical community is ready to work with the international community to conquer COVID-19. 


\section{Acknowledgements}

This work is funded by Medicine and Engineering Interdisciplinary Research Fund of Shanghai Jiao Tong University (No. YG2020YQ06), the National Key Research and Development Project (Nos. 2018YFC1705100 and 2018YFC1705103) and the National Natural Science Foundation of China (No. 71673187). We acknowledge all healthcare workers involved in the diagnosis and treatment of patients all around China. We thank National Health Commission of the People's Republic of China for coordinating data collection for patients with COVID-19.

\section{Compliance with ethics guidelines}

Shuxian Zhang, Zezhou Wang, Ruijie Chang, Huwen Wang, Chen $\mathrm{Xu}$, Xiaoyue Yu, Lhakpa Tsamlag, Yinqiao Dong, Hui Wang, and Yong Cai declare no conflicts of interest. This manuscript is a commentary and does not involve a research protocol requiring approval by the relevant institutional review board or ethics committee.

\section{References}

1. WHO. Coronavirus disease 2019 (covid-19) situation. report-47. March 7, 2020. https://www.who.int/docs/default-source/coronaviruse/situation-reports/20200307-sitrep-47-covid-19.pdf?sfvrsn = 27c364a4_2 (accessed March 8, 2020)

2. The National Health Commission of the People's Republic of China. Report on 2019- nCoV. March 8, 2020. http://en.nhc.gov.cn/ DailyBriefing.html (accessed March 8, 2020)

3. WHO. Report of the WHO-China Joint Mission on Coronavirus Disease 2019 (COVID-19). 2020. https://www.who.int/docs/ default-source/coronaviruse/who-china-joint-mission-on-covid-19final-report.pdf (accessed March 7, 2020)

4. The Health Commission of Wuhan. 41 COVID-19 patients were confirmed in Wuhan. January 11, 2020. http://www.hubei.gov.cn/ zxjy/rdhy/202001/t20200112_1910867.shtml (in Chinese) (accessed March 7, 2020)

5. Hubei Provincial Government. Notice on Wuhan novel coronavirus infection of pneumonia epidemic prevention and control. January 23, 2020. http://www.gov.cn/xinwen/2020-01/23/content_5471751. htm (in Chinese) (accessed March 7, 2020)

6. The Ministry of Finance and the National Health Commission of the People's Republic of China. China issues 9.95b yuan additional funds for public health, epidemic control. January 28, 2020. http:// en.nhc.gov.cn/2020-01/28/c_76023.htm (accessed March 7, 2020)

7. The State Council of the People's Republic of China. The notice on orderly resuming production and resuming production in enterprises. February 9, 2020. http://www.gov.cn/xinwen/2020-02/09/ content 5476550.htm (in Chinese) (accessed March 7, 2020)

8. The National Development and Reform Commission of the People's Republic of China. China's daily mask output exceeds 110 million units. March 7, 2020. http://en.nhc.gov.cn/2020-03/03/c_77257. htm (accessed March 7, 2020)

9. Wang H, Wang Z, Dong Y, Chang R, Xu C, Yu X, Zhang S, Tsamlag
L, Shang M, Huang J, Wang Y, Xu G, Shen T, Zhang X, Cai Y. Phase-adjusted estimation of the number of coronavirus disease 2019 cases in Wuhan, China. Cell Discov 2020; 6(1): 10

10. Wuhan Municipal Government.Wuhan Huoshenshan Hospital was completed today. February 2, 2020. http://www.wh.gov.cn/ 2019_web/whyw/202002/t20200202_304343.html (in Chinese) (accessed March 7, 2020)

11. Hubei Provincial Government. Wuhan Leishenshan Hospital welcomed its first confirmed 2019-nCoV patients. February 9, 2020. http://www.hubei.gov.cn/zhuanti/2020/gzxxgzbd/ys/202002/ t20200209_2021959.shtml (in Chinese) (accessed March 7, 2020)

12. The State Council. Press Conference of the joint Prevention and Control Mechanism of the State Council. March 6, 2020. http:// www.gov.cn/xinwen/gwylflkjz46/index.htm (in Chinese) (accessed March 7, 2020)

13. General Office of National Health Commission, General Office of National Administration of Traditional Chinese Medicine. Diagnostic and treatment protocol for novel coronavirus pneumonia (Trial version 7). March 4, 2020. http://www.nhc.gov.cn/xcs/ zhengcwj/202003/46c9294a7dfe4cef80dc7f5912eb1989.shtml (in Chinese) (accessed March 7, 2020)

14. Zhou P, Yang XL, Wang XG, Hu B, Zhang L, Zhang W, Si HR, Zhu Y, Li B, Huang CL, Chen HD, Chen J, Luo Y, Guo H, Jiang RD, Liu MQ, Chen Y, Shen XR, Wang X, Zheng XS, Zhao K, Chen QJ, Deng F, Liu LL, Yan B, Zhan FX, Wang YY, Xiao GF, Shi ZL. A pneumonia outbreak associated with a new coronavirus of probable bat origin. Nature 2020 Feb 3. [Epub ahead of print] doi: 10.1038/ s41586-020-2012-7

15. Zhu N, Zhang D, Wang W, Li X, Yang B, Song J, Zhao X, Huang B, Shi W, Lu R, Niu P, Zhan F, Ma X, Wang D, Xu W, Wu G, Gao GF, Tan W; China Novel Coronavirus Investigating and Research Team. A novel coronavirus from patients with pneumonia in China, 2019. N Engl J Med 2020; 382(8): 727-733

16. Lu R, Zhao X, Li J, Niu P, Yang B, Wu H, Wang W, Song H, Huang B, Zhu N, Bi Y, Ma X, Zhan F, Wang L, Hu T, Zhou H, Hu Z, Zhou W, Zhao L, Chen J, Meng Y, Wang J, Lin Y, Yuan J, Xie Z, Ma J, Liu WJ, Wang D, Xu W, Holmes EC, Gao GF, Wu G, Chen W, Shi W, Tan W. Genomic characterisation and epidemiology of 2019 novel coronavirus: implications for virus origins and receptor binding. Lancet 2020; 395(10224): 565-574

17. Huang C, Wang Y, Li X, Ren L, Zhao J, Hu Y, Zhang L, Fan G, Xu J, Gu X, Cheng Z, Yu T, Xia J, Wei Y, Wu W, Xie X, Yin W, Li H, Liu M, Xiao Y, Gao H, Guo L, Xie J, Wang G, Jiang R, Gao Z, Jin Q, Wang J, Cao B. Clinical features of patients infected with 2019 novel coronavirus in Wuhan, China. Lancet 2020; 395(10223): 497506

18. Li Q, Guan X, Wu P, Wang X, Zhou L, Tong Y, Ren R, Leung KSM, Lau EHY, Wong JY, Xing X, Xiang N, Wu Y, Li C, Chen Q, Li D, Liu T, Zhao J, Liu M, Tu W, Chen C, Jin L, Yang R, Wang Q, Zhou S, Wang R, Liu H, Luo Y, Liu Y, Shao G, Li H, Tao Z, Yang Y, Deng Z, Liu B, Ma Z, Zhang Y, Shi G, Lam TTY, Wu JTK, Gao GF, Cowling BJ, Yang B, Leung GM, Feng Z. Early transmission dynamics in Wuhan, China, of novel coronavirus-infected pneumonia. N Engl J Med 2020 Jan 29. [Epub ahead of print] doi: 10.1056/ NEJMoa2001316

19. Wu Z, McGoogan JM. Characteristics of and important lessons from the coronavirus disease 2019 (COVID-19) outbreak in China: summary of a report of 72314 cases from the Chinese Center for 
Disease Control and Prevention. JAMA 2020 Feb 24. [Epub ahead of print] doi: 10.1001/jama.2020.2648

20. Li G, De Clercq E. Therapeutic options for the 2019 novel coronavirus (2019-nCoV). Nat Rev Drug Discov 2020; 19(3): 149150

21. Tang B, Wang X, Li Q, Bragazzi NL, Tang S, Xiao Y, Wu J. Estimation of the transmission risk of the 2019-nCoV and its implication for public health interventions. J Clin Med 2020; 9(2):
462

22. Li J, Wang Y, Gilmour S, et al. Estimation of the epidemic properties of the 2019 novel coronavirus: a mathematical modeling study. medRxiv 2020; doi: 10.1101/2020.02.18.20024315

23. The Chinese Center for Disease Control and Prevention. China CDC publishes multilingual epidemic control manuals for COVID-19. March 2, 2020. http://en.nhc.gov.cn/2020-03/02/c_77204.htm (accessed March 7, 2020) 